\title{
MEASUREMENTS OF RISK IN FISHERIES MANAGEMENT
}

\author{
T.J. STEWART \\ Department of Statistical Sciences \\ University of Cape Town \\ Rondebosch 7700 \\ South Africa
}

\begin{abstract}
An important goal in many fisheries management problems is perceived to be minimization of risk. This paper examines the problem of measuring risk by means of meaningful attributes or surrogate measures, for use in multiple criteria decision support systems. It is found that exponential utility functions, which are associated in this context with geometric discounting of the future, give a poor fit in many cases relative to power functions. The implication is that conventional mean-variance measures of risk may be less appropriate than cumulative probability measures.
\end{abstract}

Keywords: risk assessment, resource management, multicriteria decision support

\section{INTRODUCTION}

One of the most controversial issues in the management of fisheries is the determination of the total allowable catch (TAC) for a particular year. Considerable effort has gone into the development and assessment of decision rules which can be applied in a systematic manner to determine these TACs for a variety of marine stocks, including not only fish, but also whales and lobsters for example. Choice of the decision rule is clearly a multiple criteria decision problem, in which clashes between socio economic and environmental goals, and between short-and longterm goals are evident. In this paper, we shall focus on one criterion which seems to emerge in virtually every study, viz. the minimization or limitation of "risk". Everyone agrees that this is an important criterion, but it is not easy to tie down what is meant by "risk" in this context. Although we focus here on fisheries management, it does 
appear that many of the concepts may carry over to other environmental management problems.

Risk undoubtedly refers in some manner to the size, timing and likelihood of an undesirable event. In the case of fisheries, this event is typically the collapse of the relevant stock (i.e. the reduction in the stock below some viable level). Note that such an event is undesirable from both economic and environmental considerations, and thus represents both economic and environmental "risk", although attitudes towards this risk (for example, risk aversion) may differ in the two cases. It is the manner in which size, timing and likelihood interact which is not well-defined. From the economic point of view, a common assumption in the financial literature is to equate risk with variance (or standard deviation) of returns. An alternative surrogate measure of risk in fisheries management may be the probability of the stock falling below some specified level within some specified time horizon, assuming that the decision rule under consideration is consistently applied throughout. In this paper, we evaluate the validity of such surrogate measures, and some implications thereof.

In the next section we report on a workshop involving fisheries scientists and fishing industry representatives, at which attempts were made to assess preferences for different probability distributions of time to occurrence of a number of undesirable events. This was followed up by a postal questionnaire involving more detailed assessments, but which only involved the scientist group. This is described in Section 3 , in which it is demonstrated that although respondents were able to answer the questions with high degree of consistency, the results are inconsistent with geometric discounting over time or constant relative risk aversion (i.e. the assumption of an exponential utility function). A power function model gives a much better fit. In the final two sections of the paper, we describe attempts at representing risk preferences by a variety of simple surrogate measures ("attributes"), and the implications of these for the design of a multicriteria decision support system for fisheries management. An indication is given there of further research which is necessary. 


\section{DIRECT VALUE JUDGEMENTS: INDUSTRY AND SCIENTIST GROUPS}

During 1992, a workshop was held, involving representatives from two major fish product companies, the SA Inshore Fishing Association and the Sea Fisheries Research Institute (SFRI) of the then Department of Environment Affairs. The SFRI group can broadly be classified as fisheries scientists, while the other participants can be viewed as representing the commercial fishing industry.

Table 1: Median-preference times (years) to undesirable events

\begin{tabular}{|l|c|c|}
\hline \multirow{2}{*}{ Event } & \multicolumn{2}{|c|}{ Group } \\
\cline { 2 - 3 } & SFRI & Industry \\
\hline Severe short-term drop in all pelagic species & $15-20$ & $20-30$ \\
Severe short-term drop in anchovy stocks & $5-10$ & $10-15$ \\
Long term drop in pilchard stocks & $20-30$ & $20-30$ \\
\hline
\end{tabular}

The first task of the workshop was to identify what were the "undesirable events" of concern in considering risks to the pelagic fishery of the Western Cape. Although a number of variations were discussed, all the events identified came down to a substantial drop in the resource and/or its availability. Variations referred to (i) specific species versus total stock; (ii) distinctions between large but short-term drops, and smaller but sustained (permanent or long-term) drops; and (iii) regional availability versus total stock size.

"In the long run, we shall all be dead"! It was recognized that if the exploitation of a fishery is continued for long enough, such undesirable events will occur eventually (through extremes of natural variation even in the absence of over-exploitation). It is meaningful, therefore, to think of the time until the undesired event first occurs, say $T$. Presumably, the longer the event can be delayed (i.e. the larger the value of $T$, the better. Two sets of questions were posed to the workshop in the light of this observation:

1. If we place a "value" of 0 on the "event" occurring within one or two years (i.e. $T$ = 1), and a value of 100 on $T>50$, what value of $T$ would correspond to a value of 50 ? (We shall term this the "median preference time".) This question was posed 
in the context of three distinct risk events. These are shown, together with the responses from the scientist and industry groups respectively, in Table 1.

Two observations can be made from the table:

- The SFRI scientists tended to select rather smaller median-preference times than did the industry, suggesting a rather higher rate of discounting of the future, contrary perhaps to what might have been anticipated. The differences are not large, however, and it is possible that there were different perceptions of the question.

- The median-preference time is substantially lower for the short-term drops in anchovy stocks than for the other events, i.e. demonstrating greater concern for the immediate future (higher levels of discounting) than for the other events. This may possibly be due to the perception of greater natural volatility in anchovy stocks.

2. The second question was phrased as follows: "Suppose that you are faced with a choice between (a) a policy in which the undesirable event occurs for sure at about the median-preference time chosen previously; or (b) a policy in which there is a probability $p$ of occurrence of this event within 1-2 years, but a probability $1-p$ that $T>50$ (with no other possibilities). How small must $p$ be, before you would prefer the second policy?" The point was that for large $p$, option (a) would undoubtedly be preferred, but that for some "sufficiently small" probability $p$, option (b), giving a high probability of almost indefinite delay until the event occurs, would ultimately be preferred.

If the values generated in response to the first question satisfied the axioms of expected utility theory, then the answer to the second question should be $p \approx 0.5$. In fact, both groups indicated very much smaller values for $p$, indicating a high degree of risk aversion relative to the directly assessed values (with the SFRI group being more risk averse, with $p$ as low as 0.05 , than the industry group with $p \approx 1 / 6$ ). Whatever the reasons for this inconsistency between directly assessed values and von Neumann-Morgenstern utilities (Keeney and Raiffa [1], Chapter 4), it is clear that 
if any average risk measure is to be computed, then it cannot be based on judgementally scaled values as above: it is essential to capture preferences between gambles in a more direct manner. This was the motivation for the study reported in the next Section.

\section{DETAILED RISK EVALUATION: SCIENTIST GROUP}

It was agreed at the workshop described in the previous section, that the workshop would be followed up by a postal survey, in which respondents would be asked to assess probabilities on various events in such a way that they would be indifferent between two outcomes. An identical set of questions was posed for each of the following "risk events":

(1) Severe short-term (1-3 year) drop in anchovy stocks and catches;

(2) Severe short-term (1-3 year) drop in total pelagic stocks and catches;

(3) Substantial and sustained long term drop in average annual yields of pilchards;

(4) Substantial and sustained long term drop in average annual yields of total pelagic species;

(5) Loss of viability of the industry;

(6) Severe changes in the regional availability of pelagic stocks.

The questions that were asked in each case are summarized in the Appendix. The questionnaires containing these questions were initially sent to all participants in the workshop, but in the end only the fisheries' scientist group returned the questionnaires.

In interpreting responses from the questionnaire, let us firstly investigate the extent to which the revealed preferences are consistent with the axioms of expected utility, based on time to occurrence of any one of the above risk events ( $T$ ) as the underlying measure of performance. Consistency with the axioms implies the existence of a real-valued function $u(T)$, such that one gamble (scenario in the questionnaire) will be preferred to another if and only if it has a higher expectation of $u(T)$. The origin and scale of such a function can be arbitrarily chosen. Let us choose to fix the origin such that $u(2)=0$ (for time measured in years). This was chosen on 
the assumption that no policy would be followed in which there was a sizeable chance of the risk event occurring in the first year; thus $T=2$ was likely to be the most unfavourable event in any comparison of management strategies, and for which respondents would be able to make any meaningful comparisons. With this as origin, we would be useful to define the scale such that $u(T) \rightarrow 1$ as $T \rightarrow \infty$. For practical purposes in analysing the results of the questionnaire, we supposed that $\mathbf{4 0}$ years was "approaching" infinity. Thus, for each pair of questions listed in Appendix: and using $u(40) \approx 1$, there is in principle sufficient information to estimate $u(7)$ and $u(15)$ for each risk event.

In practice the estimates based on the first three pairs of questions revealed a high degree of consistency (as will be described in the following paragraph), but those based on the fourth and fifth pairs were considerably less so, while those based on the last pair can only be described as highly erratic.

Tables 2 and 3 record the results of one-way analyses of variance performed on the estimates for $u(7)$ and $u(15)$ respectively, based on the first three pairs of questions, using respondent, risk event and type (i.e. pair) of question as factors. Only responses for three of the six risk events (viz. 1, 3 and 4) were included in the ANOVA: the reason for this was that the respondents tended to group the risk events, stating identical risk preferences within groups, and only these three could be separated out across all respondents. Even between these three risk events, however, the differences turned out to be not significant, indicating a strong measure of consistency in risk and time preferences across all risk events. In fact, over the two ANOVA tables, the only significant effect is the respondent effect for $u(7)$, which is significant at the $5 \%$ level, but not at the $1 \%$ level. Overall, therefore, we find a high level of consistency between estimates based on the three sets of questions. 
Table 2: ANOVA table for estimates of $u(7)$

\begin{tabular}{|l|c|c|c|c|c|}
\hline \multicolumn{1}{|c|}{$\begin{array}{c}\text { Source of } \\
\text { Variation }\end{array}$} & $\begin{array}{c}\text { Sum of } \\
\text { Squares }\end{array}$ & $\begin{array}{c}\text { Degrees of } \\
\text { Freedom }\end{array}$ & $\begin{array}{c}\text { Mean } \\
\text { Square }\end{array}$ & F-ratio & Sign level \\
\hline Respondents & 0.304 & 2 & 0.152 & 3.84 & 0.039 \\
Risk event & 0.062 & 2 & 0.031 & 0.79 & 0.470 \\
Question type & 0.249 & 2 & 0.124 & 3.14 & 0.065 \\
& & & & & \\
Residuals & 0.791 & 20 & 0.0396 & & \\
\hline
\end{tabular}

Table 3: ANOVA table for estimates of $u(15)$

\begin{tabular}{|l|c|c|c|c|c|}
\hline $\begin{array}{c}\text { Source of } \\
\text { Variation }\end{array}$ & $\begin{array}{c}\text { Sum of } \\
\text { Squares }\end{array}$ & $\begin{array}{c}\text { Degrees of } \\
\text { Freedom }\end{array}$ & $\begin{array}{c}\text { Mean } \\
\text { Square }\end{array}$ & F-ratio & Sign level \\
\hline Respondents & 0.181 & 2 & 0.091 & 2.11 & 0.150 \\
Risk event & 0.228 & 2 & 0.114 & 2.65 & 0.096 \\
Question type & 0.282 & 2 & 0.141 & 3.27 & 0.059 \\
& & & & & \\
Residuals & 0.860 & 20 & 0.0430 & & \\
\hline
\end{tabular}

Closer examination of the responses revealed that the probabilities assessed in answer to the 4th and 5 th pairs of questions were substantially smaller than those for the first three. We could conjecture that the known problems of assessing small probabilities (cf. Spetzler and Stäel von Holstein [3]) might have contributed to the inconsistencies which arose. The final pair of questions require comparative evaluation of all four times to occurrence, which must therefore be seen as the most complicated pair of questions. In a personal communication, Simon French has suggested a further complexity which might apply to the last three pairs of questions. He pointed out that the event "occurrence in $\mathbf{4 0}$ years" is probably viewed by respondents as essentially "does not occur". The first three sets of choices then involve either different levels of uncertainty regarding occurrence of the event itself, or complete certainty. The last three pairs involve (for scenario 2 in pairs 4 and 5 , and for both scenarios in the last pair) a situation in which the event itself is sure, but the timing is not. This may well have an influence on the respondents mental frames of reference, thus leading to inconsistent responses. 
Whatever the reasons for the inconsistencies in the last three pairs of questions, we can, nevertheless, conclude that it is possible to obtain estimates of the "utilities" of times to occurrence, based on simple lotteries, and which have a degree of consistency across respondents from the same interest group, provided that:

- The lotteries are indeed sufficiently "simple"; and

- The responses do not involve small probabilities (e.g. less than 0.1).

The non-significant differences between results from the first three pairs of questions, and the only marginally more significant differences between respondents, indicate a high degree of consistency. More importantly for the purposes of this paper is the lack of any significant differences between the assessments for the different risk events. This suggests a form of consistency in risk attitude from the conservation point of view which transcends differences between specific threats. In order to investigate this further, averages (across question types, threat types and respondents) were computed for $u(7)$ and $u(15)$, as a summary of the risk attitudes of the scientist group, so that these can be compared with other more conventional models. These averages were:

$$
\begin{gathered}
u(7)=0.52 \\
u(15)=0.67
\end{gathered}
$$

and are plotted as the discrete points in Figure 1. Use of these averages does not imply that the utilities of all respondents for all threats are necessarily identical: it serves rather to differentiate the consistently common features of the risk attitudes of the conservation scientists, from those implied by standard exponential utility or geometric discounting models.

Suppose, now, that we attempt to model the future in terms of some form of economic discounting. A simple model would be to assume that the catastrophic event implies a large loss, say $L$, occurring at some specific time $t$. For any given discounting factor $r(0<r<1)$, the discounted value of this loss will be $L r^{t}$. Relative 
to the worst case of an immediate loss, the value of delaying the loss until $t$ can be viewed as $L(1-r)$, which can be written in the form a - be $e^{-k t}$, where $k=-\ln r>0$. Without loss of generality this can (for purposes of comparing outcomes) be rescaled to be 0 at $t=2$ and 1 for infinitely large $t$, in which case the resultant discounted value can be expressed in the form $1-e^{-k(t-2)}$. The principle of discounting future benefits is widely accepted in most economic analyses, and it may thus be expected that we should be modelling $u(t)=1-e^{-k(t-2)}$, i.e. choosing policies so as to maximize the expectation of $u(\eta)$. This would be consistent with the phenomenon of constant relative risk aversion for time to occurrence, of. Keeney and Raiffa [1], Section 4.6 (a quite frequently invoked assumption). It turns out, however, that the least squares estimate for $k$ to give the best fit to the two values determined above is $k=0.093$, and the resulting exponential curve is displayed in Figure 1. Clearly, the estimated values are highly inconsistent with the assumption of the exponential form (and thus with conventional geometric discounting, for any discounting rate).

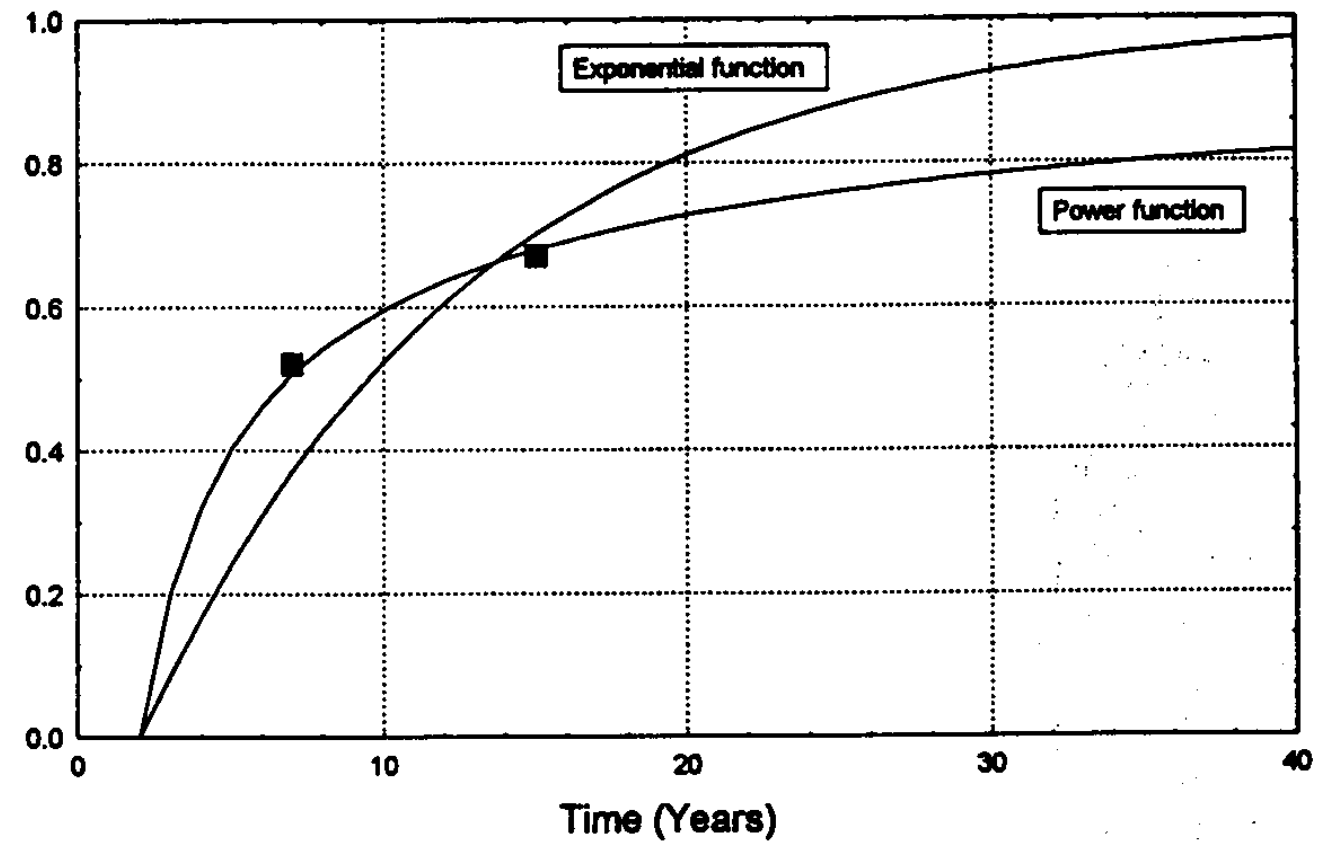

Figure 1: Utilities of times to event

Prelec and Loewenstein [2] provided a set of axioms for time preferences, and proposed a general discount function of the form $(1+\alpha t)^{-\beta / \alpha}$, as being the only 
function consistent with the given axioms. This can be expressed as a utility function on time to occurrence, scaled so that $u(2)=0$ and $u(t) \rightarrow 1$ as $t \rightarrow \infty$, of the following form:

$$
u(t)=1-\left(\frac{1+\alpha t}{1+2 \alpha}\right)^{-\beta / \alpha}
$$

This tends to the exponential function in the limit as $\alpha \rightarrow 0$, but to a function of the form $1-(2 / t)^{\gamma}$ as $\alpha \rightarrow \infty$, where $\gamma=\beta / \alpha$ (i.e. assuming that the ratio $\beta / \alpha$ remains finite as $\alpha \rightarrow \infty$. It can easily be seen that the fit to the values for $u(7)$ and $u(15)$ improves monotonically as $\alpha$ increases, so that the power function form appears more appropriate in this case. The best fit value for $\gamma$ in this case is 0.564 , and the resulting function is plotted in Figure 1. The goodness of the fit is clearly demonstrated.

The practical importance of this result is that we still do have a measure of future discounting, but that this is slower than any geometric discounting. We conjecture that this might apply to other environmental management contexts, with the implication that even arbitrarily small discount rates, if applied geometrically, will ultimately under-value consequences beyond some horizon! This appears to be an issue worthy of further research.

\section{IMPLICATIONS FOR MEASURES OF RISK}

The implication of the last section is that preferences for different times until the occurrence of identified undesirable events, from the point of view represented by the fisheries' scientists (which we shall designate as the "conservation" interest), can be represented by a "utility function" of the form $u(t)=1-(2 / t)^{\gamma}$, where the arbitrary constant terms are chosen to be consistent with the normalizations used above. On the other hand, conventional discounting (present value) analyses, which are wellaccepted and justified for financial risks (and which we thus presume to be the primary concern of the "industry" interest) has been argued in the previous section to lead to a utility function of the form $u(t)=\left(1-e^{-k t}\right) /\left(1-e^{-2 k}\right.$ ) (using the same normalization). 
In practice, it would be difficult to identify the form of utility function, and to estimate the associated parameters, for each interest group that might be represented. For the purposes of an interactive decision support system, it is usually necessary to identify a set of attributes or surrogate criteria to represent each underlying concem (in this case risk), in such a way that all users of the system can compare outcomes in terms of these attributes alone, without serious loss of discrimination. It is important, however, that the set of attributes used be as small as possible. The question thus arises: is there a parsimonious set of surrogate criteria which can capture risk preferences for all interest groups involved in the determination of TACs?

In the consideration of financial risk, it is common to use mean and variance (or standard deviation) as surrogate measures (cf. Markowitz portfolio theory). As a preliminary attempt at evaluating the usefulness of mean/variance measures, we took a very simple example of a 4-point discrete distribution on times to occurrence. The times were $3,8,13$ and 18 . If mean and variance are fixed, then this defines three linear constraints on the four probabilities, leaving one degree of freedom. It is then simple to generate probabilities uniformly on the space defined by these three constraints. Fixing the mean (arbitrarily) at 8 , simulations were conducted for three different variances, as indicated in Table 4. Each simulation consisted of 100 repetitions for the same mean and variance, and the ranges of utilities for the above functions (with $\gamma=0.564$ and $k=0.07$, a best fit to the industry time preferences revealed at the workshop) over the distributions generated were recorded, and are also displayed in the table.

Table 4: Ranges of utility values for fixed mean and variance

\begin{tabular}{|c|c|c|}
\hline Variance & $\begin{array}{c}\text { Conservation } \\
\text { (power fn.) }\end{array}$ & $\begin{array}{c}\text { Industry } \\
\text { (expon. fn.) }\end{array}$ \\
\hline 15 & $0.475-0.492$ & $0.408-0.410$ \\
20 & $0.451-0.474$ & $0.401-0.403$ \\
25 & $0.429-0.457$ & $0.393-0.397$ \\
\hline
\end{tabular}


The exponential utilities ("industry") are nearly constant for constant mean and variance, confirming the conventional wisdom. But for the power function utilities ("conservation"), the spread of utilities for fixed mean and variance is substantial (of the same order as the differences between the variance categories). In fact, for the larger two variances there is a substantial overlap, implying that there exist cases where the conservation interest would prefer a situation with the same mean and larger variance. Clearly, therefore, use of mean and variance alone as surrogate measures may be very misleading in this context.

Two alternative sets of measures come immediately to mind. They are (i) use of quantiles of the distribution of $T$ (e.g. upper and lower quartiles); and (ii) use of cumulative probabilities up to certain fixed periods (e.g. probabilities of $T<3$ and of $T>10$ respectively). We report here on some preliminary simulation studies aimed at evaluating these alternatives. In these studies, we set $T=X+1$, where $X$ was distributed according to a mixture of Poisson distributions, i.e. with the probability function of $X$ given by:

$$
\alpha \frac{\mu^{x} e^{-\mu}}{x !}+(1-a) \frac{\lambda^{x}}{x !} e^{-\lambda}
$$

for $x=0,1, \ldots$ Values of $\alpha, \mu$ and $\lambda$ were generated uniformly over the intervals [0.1$0.4],[3-8]$ and [10-20] respectively, to create a number of randomly generated cases (hypothetical policy alternatives). In each repetition, the expected utilities for the above two functional forms, the mean and standard deviation, the upper and lower quartiles, and cumulative probabilities to $T=3$ and $T=10$ were calculated. Subsequently, and based on 50 simulated cases, the true expected utilities were regressed against each pair of measures (mean and standard deviation; upper and lower quartiles; and the two cumulative probabilities), and the multiple correlations $\left(R^{2}\right)$ calculated in each case. These are recorded in Table 5. The multiple correlation coefficients can, however, give a misleadingly optimistic picture in this context. The $\left(R^{2}\right)$ is, for example, inflated in the simulation results by cases which are either so obviously good, or so obviously poor, that any sensible measure will flag these accordingly. In practice, however, the surrogate measures are to be used for rank ordering a set of policies, from which obviously poor options have been deleted 
(while if there is an obvious clear winner, no further analysis is necessary). In such cases, any $R^{2}$ which differs from 1 by any substantial amount could still imply a rank ordering very different from what it should be. In order to give some feel for this important issue, Table 5 also includes the Spearman rank correlations between the true orderings (according to the assumed utility functions for each interest), and those predicted from each pair of measures (based on the estimated regression coefficients), based on the top 25 of the 50 simulated cases, after deleting the best alternative (which had clearly dominated all other cases).

Table 5: Correlations between utilities and alternative surrogate measures

\begin{tabular}{|l|c|c|c|c|}
\hline \multirow{2}{*}{ Predictor } & \multicolumn{2}{c|}{ Conservation } & \multicolumn{2}{c|}{ Industry } \\
\cline { 2 - 5 } & $R^{2}$ & Rank Corr. & $R^{2}$ & Rank Corr. \\
\hline MeanNariance & 0.923 & 0.78 & 0.990 & 0.99 \\
Quartiles & 0.801 & 0.69 & 0.953 & 0.96 \\
Cum.Probs. & 0.968 & 0.91 & 0.912 & 0.77 \\
\hline
\end{tabular}

Once again, for the exponential function (which we have designated the "industry" interest), the mean and standard deviation is an excellent surrogate measure of risk. For the conservation interests (power function utility), the mean and standard deviation is far less satisfactory. The use of quartiles is even worse, but there appears to be a prima facie case to be made that the use of two well-chosen cumulative probabilites may be useful. These results must be seen as preliminary, as considerable scope exists for "fine-tuning" the choice of points at which probabilities are to be reported as surrogate measures. It seems inevitable, however, that the best choice of these will depend on the prior expectation of ranges of distribution which could occur: perhaps the choice of points will have to be based on selected quantiles of some form of "prior" distribution of time to occurrence. These items will be addressed in follow-up research.

\section{IMPLICATIONS FOR DECISION SUPPORT SYSTEMS}

In other unpublished studies with which we have been involved, it has frequently emerged that decision support systems for fisheries' management need to incorporate some form of multiple criteria decision aid, based on criteria representing 
long term average yield, inter-annual variations in yield, risk and environmental impact. The first two criteria are naturally quantifiable, and in the companion paper we suggest some means of assessing environmental impact based on work done by us in water management (Stewart and Scott [4]). The work reported in the present paper thus completes the picture. It appears that it may be necessary at this stage to include four risk "attributes", viz. mean, variance (or standard deviation), and two cumulative probabilities for time until some specified risk event (which would be context dependent). It is possible, however, that further investigation may allow us to reduce these four attributes to just two or three cumulative probabilities.

The importance of this result is that it allows the decision support system to utilize relatively simple multiple criteria decision aiding procedures such as additive value functions, interactive goal programming or outranking (ELECTRE).

\section{REFERENCES}

[1] Keeney, R.L. and Raiffa, H. Decisions with Multiple Objectives, Wiley, New York (1976)

[2] Prelec, D. and Loewenstein, G. "Decision making over time and uncertainty: a common approach", Management Science, 37, 770-786 (1991)

[3] Spetzler, C.S. and C-A S Stäel von Holstein, "Probability encoding in decision analysis", Management Science, 22, 340-358 (1975)

[4] Stewart, T.J. and Scott, L. "A scenario-based framework for multicriteria decision analysis in water resources planning", Water Resources Research, 31, 2835-2843 (1995) 


\section{APPENDIX: QUESTIONS ASKED IN POSTAL SURVEY}

For each risk event, the respondents were asked to select the probability $p$ in each of the following 12 questions (grouped into six pairs) which would make the two scenarios of approximately equal desirability:

\begin{tabular}{|l|l|l|}
\hline \multicolumn{1}{|c|}{ Scenario 1} & \multicolumn{1}{|c|}{ Scenario 2} \\
\hline 1 & Occurrence in 7 years for sure & $\begin{array}{l}\text { Occurrence in } 2 \text { years with probability } p \\
\text { or in } 40 \text { years otherwise }\end{array}$ \\
\hline 2 & Occurrence in 15 years for sure & $\begin{array}{l}\text { Occurrence in } 2 \text { years with probability } p \\
\text { or in } 40 \text { years otherwise }\end{array}$ \\
\hline 3 & $\begin{array}{l}\text { Occurrence in } 2 \text { years with probability } \\
0.05 \text { or in } 40 \text { years otherwise }\end{array}$ & $\begin{array}{l}\text { Occurrence in } 7 \text { years with probability } p \\
\text { or in } 40 \text { years otherwise }\end{array}$ \\
\hline 4 & $\begin{array}{l}\text { Occurrence in } 2 \text { years with probability } \\
0.05 \text { or in } 40 \text { years otherwise }\end{array}$ & $\begin{array}{l}\text { Occurrence in } 15 \text { years with probability } \\
p \text { or in } 40 \text { years otherwise }\end{array}$ \\
\hline 6 & $\begin{array}{l}\text { Occurrence in } 2 \text { years with probability } \\
0.2 \text { or in } 40 \text { years otherwise }\end{array}$ & $\begin{array}{l}\text { Occurrence in } 7 \text { years with probability } p \\
\text { or in } 40 \text { years otherwise }\end{array}$ \\
\hline 7 & $\begin{array}{l}\text { Occurrence in } 2 \text { years with probability } \\
0.05 \text { or in } 40 \text { years otherwise }\end{array}$ & $\begin{array}{l}\text { Occurrence in } 2 \text { years with probability } p \\
\text { or in } 7 \text { years otherwise }\end{array}$ \\
\hline 8 & $\begin{array}{l}\text { Occurrence in } 2 \text { years with probability } \\
0.05 \text { or in } 40 \text { years otherwise }\end{array}$ & $\begin{array}{l}\text { Occurrence in } 2 \text { years with probability } p \\
\text { or in } 15 \text { years otherwise }\end{array}$ \\
\hline 9 & $\begin{array}{l}\text { Occurrence in } 2 \text { years with probability } \\
0.2 \text { or in } 40 \text { years otherwise }\end{array}$ & $\begin{array}{l}\text { Occurrence in } 2 \text { years with probability } p \\
\text { or in } 7 \text { years otherwise }\end{array}$ \\
\hline 10 & $\begin{array}{l}\text { Occurrence in } 2 \text { years with probability } \\
0.2 \text { or in } 40 \text { years otherwise }\end{array}$ & $\begin{array}{l}\text { Occurrence in } 2 \text { years with probability } p \\
\text { or in } 15 \text { years otherwise }\end{array}$ \\
\hline 11 & $\begin{array}{l}\text { Occurrence in } 2 \text { years with probability } \\
0.05 \text { or in } 15 \text { years otherwise }\end{array}$ & $\begin{array}{l}\text { Occurrence in } 7 \text { years with probability } p \\
\text { or in } 40 \text { years otherwise }\end{array}$ \\
\hline 12 & $\begin{array}{l}\text { Occurrence in } 2 \text { years with probability } \\
0.2 \text { or in } 15 \text { years otherwise }\end{array}$ & $\begin{array}{l}\text { Occurrence in } 7 \text { years with probability } p \\
\text { or in } 40 \text { years otherwise }\end{array}$ \\
\hline
\end{tabular}

